\title{
A Perturbed Ostrowski-Type Inequality on Time Scales for $k$ Points for Functions Whose Second Derivatives Are Bounded
}

\author{
Wenjun Liu, ${ }^{1}$ Quốc Anh Ngôo, ${ }^{2,3}$ and Wenbing Chen ${ }^{1}$ \\ ${ }^{1}$ College of Mathematics and Physics, Nanjing University of Information Science and Technology, \\ Nanjing 210044, China \\ ${ }^{2}$ Department of Mathematics, College of Science, Viet Nam National University, Hanoi, Vietnam \\ ${ }^{3}$ Department of Mathematics, National University of Singapore, 2 Science Drive 2, Singapore 117543
}

Correspondence should be addressed to Wenjun Liu, wjliu@nuist.edu.cn

Received 6 May 2008; Accepted 13 August 2008

Recommended by Kunquan Lan

We first derive a perturbed Ostrowski-type inequality on time scales for $k$ points for functions whose second derivatives are bounded and then unify corresponding continuous and discrete versions. We also point out some particular perturbed integral inequalities on time scales for functions whose second derivatives are bounded as special cases.

Copyright (c) 2008 Wenjun Liu et al. This is an open access article distributed under the Creative Commons Attribution License, which permits unrestricted use, distribution, and reproduction in any medium, provided the original work is properly cited.

\section{Introduction}

The following integral inequality which was first proved by Ostrowski in 1938 has received considerable attention from many researchers [1-9].

Theorem 1.1. Let $f:[a, b] \rightarrow \mathbb{R}$ be continuous on $[a, b]$ and differentiable in $(a, b)$ and its derivative $f^{\prime}:(a, b) \rightarrow \mathbb{R}$ is bounded on $(a, b)$, that is, $\left\|f^{\prime}\right\|_{\infty}:=\sup _{t \in(a, b)}\left|f^{\prime}(x)\right|<\infty$. Then for any $x \in[a, b]$, the following inequality holds:

$$
\left|f(x)-\frac{1}{b-a} \int_{a}^{b} f(t) d t\right| \leq\left(\frac{1}{4}+\frac{(x-(a+b) / 2)^{2}}{(b-a)^{2}}\right)(b-a)\left\|f^{\prime}\right\|_{\infty} .
$$

The inequality is sharp in the sense that the constant $1 / 4$ cannot be replaced by a smaller one.

In 1988, Hilger [10] developed the theory of time scales as a theory capable to contain both difference and differential calculus in a consistent way. Since then, many authors have 
studied the theory of certain integral inequalities on time scales. For example, we refer the reader to [11-18]. In [15], Bohner and Matthews established the following so-called Ostrowski inequality on time scales.

Theorem 1.2 (see [15, Theorem 3.5]). Let $a, b, x, t \in \mathbb{T}, a<b$, and $f:[a, b] \rightarrow \mathbb{R}$ be differentiable. Then

$$
\left|\int_{a}^{b} f^{\sigma}(t) \Delta t-f(x)(b-a)\right| \leq M\left(h_{2}(x, a)+h_{2}(x, b)\right)
$$

where $h_{k}(\cdot, \cdot)$ is defined by Definition 2.9 below and $M=\sup _{a<x<b}\left|f^{\Delta}(x)\right|$. This inequality is sharp in the sense that the right-hand side of (1.1) cannot be replaced by a smaller one.

Liu and Ngô then generalize the above Ostrowski inequality on time scales for $k$ points $x_{1}, x_{2}, \ldots, x_{k}$ in [19]. They also extended the result by considering functions whose second derivatives are bounded in [20]. They obtained the following theorem.

Theorem 1.3. Let $a, b, x, t \in \mathbb{T}, a<b$, and $f:[a, b] \rightarrow \mathbb{R}$ be a twice differentiable function on $(a, b)$ and $f^{\Delta \Delta}:(a, b) \rightarrow \mathbb{R}$ bounded, that is, $M:=\sup _{a<t<b}\left|f^{\Delta \Delta}(x)\right|<\infty$. Then

$$
\left|\int_{a}^{b} f^{\sigma}(t) \Delta t-f^{\sigma}(x)(b-a)+\left(h_{2}(x, a)-h_{2}(x, b)\right) f^{\Delta}(x)\right| \leq M\left(h_{3}(x, a)-h_{3}(x, b)\right) .
$$

Theorem 1.3 may be thought of as a perturbed version of Theorem 1.2. In the present paper we will first derive a perturbed Ostrowski-type inequality on time scales for $k$ points $x_{1}, x_{2}, \ldots, x_{k}$ for functions whose second derivatives are bounded and then unify corresponding continuous and discrete versions. We also point out some particular perturbed integral inequalities on time scales for functions whose second derivatives are bounded as special cases.

\section{Time scales essentials}

In this section, we briefly introduce the time scales theory and refer the reader to Hilger [10] and the books [21-23] for further details (see also $[19,20]$ ).

Definition 2.1. A time scale $\mathbb{T}$ is an arbitrary nonempty closed subset of the real numbers.

Definition 2.2. For $t \in \mathbb{T}$, one defines the forward jump operator $\sigma: \mathbb{T} \rightarrow \mathbb{T}$ by $\sigma(t)=\inf \{s \in \mathbb{T}$ : $s>t\}$, while the backward jump operator $\rho: \mathbb{T} \rightarrow \mathbb{T}$ is defined by $\rho(t)=\sup \{s \in \mathbb{T}: s<t\}$.

In this definition, we put inf $\varnothing=\sup \mathbb{T}$ (i.e., $\sigma(t)=t$ if $\mathbb{T}$ has a maximum $t$ ) and $\sup \varnothing=\inf \mathbb{T}$ (i.e., $\rho(t)=t$ if $\mathbb{T}$ has a minimum $t$ ), where $\varnothing$ denotes the empty set. If $\sigma(t)>t$, then we say that $t$ is right-scattered, while if $\rho(t)<t$, then we say that $t$ is left-scattered. Points that are right-scattered and left-scattered at the same time are called isolated. If $\sigma(t)=t$ and $t<\sup \mathbb{T}$, then $t$ is called right dense, and if $\rho(t)=t$ and $t>\inf \mathbb{T}$, then $t$ is called left dense. Points that are both right dense and left dense are called dense. 
Definition 2.3. Let $t \in \mathbb{T}$, then two mappings $\mu, v: \mathbb{T} \rightarrow[0,+\infty)$ satisfying

$$
\mu(t):=\sigma(t)-t, \quad v(t):=t-\rho(t)
$$

are called the graininess functions.

We now introduce the set $\mathbb{T}^{\kappa}$ which is derived from the time scales $\mathbb{T}$ as follows. If $\mathbb{T}$ has a left-scattered maximum $t$, then $\mathbb{T}^{\kappa}:=\mathbb{T}-\{t\}$, otherwise $\mathbb{T}^{\kappa}:=\mathbb{T}$. Furthermore, for a function $f: \mathbb{T} \rightarrow \mathbb{R}$, we define the function $f^{\sigma}: \mathbb{T} \rightarrow \mathbb{R}$ by $f^{\sigma}(t)=f(\sigma(t))$ for all $t \in \mathbb{T}$.

Definition 2.4. Let $f: \mathbb{T} \rightarrow \mathbb{R}$ be a function on time scales. Then for $t \in \mathbb{T}^{\kappa}$, one defines $f^{\Delta}(t)$ to be the number, if one exists, such that for all $\varepsilon>0$ there is a neighborhood $U$ of $t$ such that for all $s \in U$

$$
\left|f^{\sigma}(t)-f(s)-f^{\Delta}(t)(\sigma(t)-s)\right| \leq \varepsilon|\sigma(t)-s| .
$$

We say that $f$ is $\Delta$-differentiable on $\mathbb{T}^{\kappa}$ provided $f^{\Delta}(t)$ exists for all $t \in \mathbb{T}^{\kappa}$. We talk about the second derivative $f^{\Delta \Delta}$ provided $f^{\Delta}$ is differentiable on $\mathbb{T}^{\kappa^{2}}=\left(\mathbb{T}^{\kappa}\right)^{\kappa}$ with derivative $f^{\Delta \Delta}=$ $\left(f^{\Delta}\right)^{\Delta}: \mathbb{T}^{\kappa^{2}} \rightarrow \mathbb{R}$.

Definition 2.5. A mapping $f: \mathbb{T} \rightarrow \mathbb{R}$ is called $r d$-continuous (denoted by $f \in C_{\mathrm{rd}}$ ) provided that it satisfies

(1) $f$ is continuous at each right-dense point of $\mathbb{T}$;

(2) the left-sided $\operatorname{limit}_{\lim _{s \rightarrow t-}} f(s)=f(t-)$ exists at each left-dense point $t$ of $\mathbb{T}$.

Remark 2.6. It follows from Theorem 1.74 of Bohner and Peterson [21] that every rdcontinuous function has an antiderivative.

Definition 2.7. A function $F: \mathbb{T} \rightarrow \mathbb{R}$ is called a $\Delta$-antiderivative of $f: \mathbb{T} \rightarrow \mathbb{R}$ provided $F^{\Delta}(t)=f(t)$ holds for all $t \in \mathbb{T}^{\kappa}$. Then the $\Delta$-integral of $f$ is defined by

$$
\int_{a}^{b} f(t) \Delta t=F(b)-F(a)
$$

Proposition 2.8. Let $f, g$ be $r d$-continuous, $a, b, c \in \mathbb{T}$, and $\alpha, \beta \in \mathbb{R}$. Then

(1) $\int_{a}^{b}(\alpha f(t)+\beta g(t)) \Delta t=\alpha \int_{a}^{b} f(t) \Delta t+\beta \int_{a}^{b} g(t) \Delta t$,

(2) $\int_{a}^{b} f(t) \Delta t=-\int_{b}^{a} f(t) \Delta t$,

(3) $\int_{a}^{b} f(t) \Delta t=\int_{a}^{c} f(t) \Delta t+\int_{c}^{b} f(t) \Delta t$,

(4) $\int_{a}^{b} f(t) g^{\Delta}(t) \Delta t=(f g)(b)-(f g)(a)-\int_{a}^{b} f^{\Delta}(t) g(\sigma(t)) \Delta t$,

(5) $\int_{a}^{a} f(t) \Delta t=0$,

(6) If $f(t) \geq 0$ for all $a \leq t<b$ then $\int_{a}^{b} f(t) \Delta t \geq 0$. 
Definition 2.9. Let $h_{k}: \mathbb{T}^{2} \rightarrow \mathbb{R}, k \in \mathbb{N}_{0}$ be defined by

$$
h_{0}(t, s)=1 \quad \forall s, t \in \mathbb{T}
$$

and then recursively by

$$
h_{k+1}(t, s)=\int_{s}^{t} h_{k}(\tau, s) \Delta \tau \quad \forall s, t \in \mathbb{T}
$$

Remark 2.10. It follows from Proposition 2.8(6) that if $s \leq t$, then $h_{k+1}(t, s) \geq 0$ for all $t, s \in \mathbb{T}$ and all $k \in \mathbb{N}$.

Remark 2.11. If we let $h_{k}^{\Delta}(t, s)$ denote for each fixed $s$ the derivative of $h_{k}(t, s)$ with respect to $t$, then

$$
h_{k}^{\Delta}(t, s)=h_{k-1}(t, s), \quad \text { for } k \in \mathbb{N}, t \in \mathbb{T}^{\kappa}
$$

\section{The perturbed Ostrowski inequality on time scales}

Our main result reads as follows .

Theorem 3.1. Suppose that

(1) $a, b \in \mathbb{T}, I_{k}: a=x_{0}<x_{1}<\cdots<x_{k-1}<x_{k}=b$ is a division of the interval $[a, b]$ for $x_{0}, x_{1}, \ldots, x_{k} \in \mathbb{T}$;

(2) $\alpha_{i} \in \mathbb{T}(i=0, \ldots, k+1)$ is " $k+2$ " points so that $\alpha_{0}=a, \alpha_{i} \in\left[x_{i-1}, x_{i}\right](i=1, \ldots, k)$ and $\alpha_{k+1}=b$;

(3) $f:[a, b] \rightarrow \mathbb{R}$ is a twice differentiable function on $(a, b)$ and $f^{\Delta \Delta}:(a, b) \rightarrow \mathbb{R}$ is bounded, that is, $M:=\sup _{a<t<b}\left|f^{\Delta \Delta}(t)\right|<\infty$.

Then

$$
\begin{aligned}
& \left|\int_{a}^{b} f^{\sigma}(t) \Delta t-\sum_{i=0}^{k}\left(\alpha_{i+1}-\alpha_{i}\right) f^{\sigma}\left(x_{i}\right)+\sum_{i=0}^{k-1}\left(h_{2}\left(x_{i+1}, \alpha_{i+1}\right) f^{\Delta}\left(x_{i+1}\right)-h_{2}\left(x_{i}, \alpha_{i+1}\right) f^{\Delta}\left(x_{i}\right)\right)\right| \\
& \quad \leq M \sum_{i=0}^{k-1}\left(h_{3}\left(x_{i+1}, \alpha_{i+1}\right)-h_{3}\left(x_{i}, \alpha_{i+1}\right)\right) .
\end{aligned}
$$

To prove Theorem 3.1, we need the following generalized montgomery identity for twice differentiable function. This is motivated by the ideas of Sofo and Dragomir in [24], where the continuous version of a perturbed Ostrowski inequality for twice differentiable mappings was proved. 
Wenjun Liu et al.

Lemma 3.2 (generalized montgomery identity). Under the assumptions of Theorem 3.1,

$$
\begin{aligned}
\sum_{i=0}^{k}\left(\alpha_{i+1}-\alpha_{i}\right) f^{\sigma}\left(x_{i}\right)= & \int_{a}^{b} f^{\sigma}(t) \Delta t-\int_{a}^{b} K\left(t, I_{k}\right) f^{\Delta \Delta} \Delta t \\
& +\sum_{i=0}^{k-1}\left(h_{2}\left(x_{i+1}, \alpha_{i+1}\right) f^{\Delta}\left(x_{i+1}\right)-h_{2}\left(x_{i}, \alpha_{i+1}\right) f^{\Delta}\left(x_{i}\right)\right),
\end{aligned}
$$

where

$$
K\left(t, I_{k}\right)=\left\{\begin{array}{cl}
h_{2}\left(t, \alpha_{1}\right), & t \in\left[a, x_{1}\right), \\
h_{2}\left(t, \alpha_{2}\right), & t \in\left[x_{1}, x_{2}\right), \\
\vdots & \\
h_{2}\left(t, \alpha_{k-1}\right), & t \in\left[x_{k-2}, x_{k-1}\right), \\
h_{2}\left(t, \alpha_{k}\right), & t \in\left[x_{k-1}, b\right] .
\end{array}\right.
$$

Proof. Integrating by parts and applying Proposition 2.8(4), we have

$$
\begin{aligned}
\int_{a}^{b} K\left(t, I_{k}\right) f^{\Delta \Delta}(t) \Delta t \\
=\sum_{i=0}^{k-1} \int_{x_{i}}^{x_{i+1}} K\left(t, I_{k}\right) f^{\Delta \Delta}(t) \Delta t \\
=\sum_{i=0}^{k-1} \int_{x_{i}}^{x_{i+1}} h_{2}\left(t, \alpha_{i+1}\right) f^{\Delta \Delta}(t) \Delta t \\
=\sum_{i=0}^{k-1}\left(\int_{x_{i}}^{\alpha_{i+1}} h_{2}\left(t, \alpha_{i+1}\right) f^{\Delta \Delta}(t) \Delta t+\int_{\alpha_{i+1}}^{x_{i+1}} h_{2}\left(t, \alpha_{i+1}\right) f^{\Delta \Delta}(t) \Delta t\right) \\
=\sum_{i=0}^{k-1}\left(h_{2}\left(\alpha_{i+1}, \alpha_{i+1}\right) f^{\Delta}\left(\alpha_{i+1}\right)-h_{2}\left(x_{i}, \alpha_{i+1}\right) f^{\Delta}\left(x_{i}\right)-\int_{x_{i}}^{\alpha_{i+1}} f^{\Delta}(\sigma(t)) h_{2}^{\Delta}\left(t, \alpha_{i+1}\right) \Delta t\right. \\
\left.\quad+h_{2}\left(x_{i+1}, \alpha_{i+1}\right) f^{\Delta}\left(x_{i+1}\right)-h_{2}\left(\alpha_{i+1}, \alpha_{i+1}\right) f^{\Delta}\left(\alpha_{i+1}\right)-\int_{\alpha_{i+1}}^{x_{i+1}} f^{\Delta}(\sigma(t)) h_{2}^{\Delta}\left(t, \alpha_{i+1}\right) \Delta t\right) \\
=\sum_{i=0}^{k-1}\left(h_{2}\left(x_{i+1}, \alpha_{i+1}\right) f^{\Delta}\left(x_{i+1}\right)-h_{2}\left(x_{i}, \alpha_{i+1}\right) f^{\Delta}\left(x_{i}\right)\right. \\
\left.\quad \quad-\int_{x_{i}}^{\alpha_{i+1}} f^{\Delta}(\sigma(t))\left(t-\alpha_{i+1}\right) \Delta t-\int_{\alpha_{i+1}}^{x_{i+1}} f^{\Delta}(\sigma(t))\left(t-\alpha_{i+1}\right) \Delta t\right) \\
\left.\quad+\int_{x_{1}}^{\alpha_{i+1}} f^{\sigma}(t) \Delta t-f^{\sigma}\left(x_{i+1}\right)\left(x_{i+1}-\alpha_{i+1}\right)+\int_{\alpha_{i+1}}^{x_{i+1}} f^{\sigma}(t) \Delta t\right) \\
\quad \sum_{i=0}\left(h_{2}\left(x_{i+1}, \alpha_{i+1}\right) f^{\Delta}\left(x_{i+1}\right)-h_{2}\left(x_{i}, \alpha_{i+1}\right) f^{\Delta}\left(x_{i}\right)+f^{\sigma}\left(x_{i}\right)\left(x_{i}-\alpha_{i+1}\right)\right.
\end{aligned}
$$




$$
\begin{aligned}
= & \int_{a}^{b} f^{\sigma}(t) \Delta t+\sum_{i=0}^{k-1}\left(h_{2}\left(x_{i+1}, \alpha_{i+1}\right) f^{\Delta}\left(x_{i+1}\right)-h_{2}\left(x_{i}, \alpha_{i+1}\right) f^{\Delta}\left(x_{i}\right)\right)+f^{\sigma}(a)\left(a-\alpha_{1}\right) \\
& +\sum_{i=1}^{k-1} f^{\sigma}\left(x_{i}\right)\left(x_{i}-\alpha_{i+1}\right)-f^{\sigma}(b)\left(b-\alpha_{k}\right)-\sum_{i=0}^{k-2} f^{\sigma}\left(x_{i+1}\right)\left(x_{i+1}-\alpha_{i+1}\right) \\
= & \int_{a}^{b} f^{\sigma}(t) \Delta t-\sum_{i=0}^{k} f^{\sigma}\left(x_{i}\right)\left(\alpha_{i+1}-\alpha_{i}\right)+\sum_{i=0}^{k-1}\left(h_{2}\left(x_{i+1}, \alpha_{i+1}\right) f^{\Delta}\left(x_{i+1}\right)-h_{2}\left(x_{i}, \alpha_{i+1}\right) f^{\Delta}\left(x_{i}\right)\right),
\end{aligned}
$$

that is, (3.2) holds.

Proof of Theorem 3.1. By applying Lemma 3.2, we get

$$
\begin{aligned}
& \left|\int_{a}^{b} f^{\sigma}(t) \Delta t-\sum_{i=0}^{k}\left(\alpha_{i+1}-\alpha_{i}\right) f^{\sigma}\left(x_{i}\right)+\sum_{i=0}^{k-1}\left(h_{2}\left(x_{i+1}, \alpha_{i+1}\right) f^{\Delta}\left(x_{i+1}\right)-h_{2}\left(x_{i}, \alpha_{i+1}\right) f^{\Delta}\left(x_{i}\right)\right)\right| \\
& \quad=\left|\int_{a}^{b} K\left(t, I_{k}\right) f^{\Delta \Delta}(t) \Delta t\right|=\left|\sum_{i=0}^{k-1} \int_{x_{i}}^{x_{i+1}} K\left(t, I_{k}\right) f^{\Delta \Delta}(t) \Delta t\right| \\
& \quad \leq \sum_{i=0}^{k-1} \int_{x_{i}}^{x_{i+1}}\left|K\left(t, I_{k}\right)\right|\left|f^{\Delta \Delta}(t)\right| \Delta t \leq M \sum_{i=0}^{k-1} \int_{x_{i}}^{x_{i+1}}\left|h_{2}\left(t, \alpha_{i+1}\right)\right| \Delta t \\
& \quad=M \sum_{i=0}^{k-1}\left(\int_{x_{i}}^{\alpha_{i+1}}\left(\int_{t}^{\alpha_{i+1}}\left(\alpha_{i+1}-\tau\right) \Delta \tau\right) \Delta t+\int_{\alpha_{i+1}}^{x_{i+1}} h_{2}\left(t, \alpha_{i+1}\right) \Delta t\right) \\
& \quad=M \sum_{i=0}^{k-1}\left(\int_{x_{i}}^{\alpha_{i+1}} h_{2}\left(t, \alpha_{i+1}\right) \Delta t+\int_{\alpha_{i+1}}^{x_{i+1}} h_{2}\left(t, \alpha_{i+1}\right) \Delta t\right) \\
& \quad=M \sum_{i=0}^{k-1}\left(h_{3}\left(x_{i+1}, \alpha_{i+1}\right)-h_{3}\left(x_{i}, \alpha_{i+1}\right)\right) .
\end{aligned}
$$

The proof is complete.

If we apply the the inequality (3.1) to different time scales, we will get some wellknown and some new results.

Corollary 3.3 (continuous case). Let $\mathbb{T}=\mathbb{R}$. Then our delta integral is the usual Riemann integral from calculus. Hence,

$$
h_{2}(t, s)=\frac{(t-s)^{2}}{2}, \quad \forall t, s \in \mathbb{R}
$$


Wenjun Liu et al.

This leads us to state the following inequality:

$$
\begin{aligned}
& \left|\int_{a}^{b} f(t) \Delta t-\sum_{i=0}^{k}\left(\alpha_{i+1}-\alpha_{i}\right) f\left(x_{i}\right)+\frac{1}{2} \sum_{i=0}^{k-1}\left(\left(x_{i+1}-\alpha_{i+1}\right)^{2} f^{\prime}\left(x_{i+1}\right)-\left(x_{i}-\alpha_{i+1}\right)^{2} f^{\prime}\left(x_{i}\right)\right)\right| \\
& \quad \leq \frac{M}{6} \sum_{i=0}^{k-1}\left(\left(x_{i+1}-\alpha_{i+1}\right)^{3}-\left(x_{i}-\alpha_{i+1}\right)^{3}\right),
\end{aligned}
$$

where $M=\sup _{a<x<b}\left|f^{\prime \prime}(x)\right|$.

Remark 3.4. The inequality (3.7) is exactly the generalized Ostrowski inequality shown in [24].

Corollary 3.5 (discrete case). Let $\mathbb{T}=\mathbb{Z}, a=0, b=n$. Suppose that

(1) $I_{k}: 0=j_{0}<j_{1}<\cdots<j_{k-1}<j_{k}=n$ is a division of $[0, n] \cap \mathbb{Z}$ for $j_{0}, k_{1}, \ldots, j_{k} \in \mathbb{Z}$;

(2) $p_{i} \in \mathbb{Z}(i=0, \ldots, k+1)$ is " $k+2$ " points so that $p_{0}=0, p_{i} \in\left[j_{i-1}, j_{i}\right] \cap \mathbb{Z}(i=1, \ldots, k)$ and $p_{k+1}=n$;

(3) $f(k)=x_{k}$.

Then,

$$
\begin{aligned}
& \left|\sum_{j=1}^{n} x_{j}-\sum_{i=0}^{k}\left(p_{i+1}-p_{i}\right) x_{j_{i}+1}+\sum_{i=0}^{k-1}\left(h_{2}\left(j_{i+1}, p_{i+1}\right) \Delta x_{j_{i}+1}-h_{2}\left(j_{i}, p_{i+1}\right) \Delta x_{j_{i}}\right)\right| \\
& \quad \leq M \sum_{i=0}^{k-1}\left(h_{3}\left(j_{i+1}, p_{i+1}\right)-h_{3}\left(j_{i}, p_{i+1}\right)\right)
\end{aligned}
$$

for all $i=\overline{1, n}$, where

$$
M=\sup _{1 \leq i \leq n-1}\left|\Delta^{2} x_{i}\right|, \quad h_{k}(t, s)=\left(\begin{array}{c}
t-s \\
k
\end{array}\right)
$$

for all $t, s \in \mathbb{Z}$.

Corollary 3.6 (quantum calculus case). Let $\mathbb{T}=q^{\mathbb{N}_{0}}, q>1, a=q^{m}, b=q^{n}$ with $m<n$. Suppose that

(1) $I_{k}: q^{m}=q^{j_{0}}<q^{j_{1}}<\cdots<q^{j_{k-1}}<q^{j_{k}}=q^{n}$ is a division of $\left[q^{m}, q^{n}\right] \cap q^{\mathbb{N}_{0}}$ for $j_{0}, j_{1}, \ldots, j_{k} \in$ $\mathbb{N}_{0}$;

(2) $q^{p_{i}} \in q^{\mathbb{N}_{0}}(i=0, \ldots, k+1)$ is " $k+2$ " points so that $q^{p_{0}}=q^{m}, q^{p_{i}} \in\left[q^{j_{i-1}}, q^{j_{i}}\right] \cap q^{\mathbb{N}_{0}}(i=$ $1, \ldots, k)$ and $q^{p_{k+1}}=q^{m}$

(3) $f:\left[q^{m}, q^{n}\right] \rightarrow \mathbb{R}$ is differentiable. 
Then,

$$
\begin{aligned}
& \mid \int_{q^{m}}^{q^{n}} f(q t) \Delta t-\sum_{i=0}^{k}\left(q^{p_{i+1}}-q^{p_{i}}\right) f\left(q^{j_{i}+1}\right) \\
& \quad+\sum_{i=0}^{k-1}\left(h_{2}\left(q^{j_{i+1}}, q^{p_{i+1}}\right) \frac{f\left(q^{j_{i+1}+1}\right)-f\left(q^{j_{i+1}}\right)}{(q-1) q^{j_{i+1}}}-h_{2}\left(q^{j_{i}}, q^{p_{i}}\right) \frac{f\left(q^{j_{i}+1}\right)-f\left(q^{j_{i}}\right)}{(q-1) q^{j_{i}}}\right) \mid \\
& \leq M \sum_{i=0}^{k-1}\left(h_{3}\left(q^{j_{i+1}}, q^{p_{i+1}}\right)-h_{3}\left(q^{j_{i}}, q^{p_{i+1}}\right)\right)
\end{aligned}
$$

where

$$
M=\sup _{q^{m}<t<q^{n}}\left|\frac{f\left(q^{2} t\right)-(q+1) f(q t)+q f(t)}{q(q-1)^{2} t^{2}}\right|, \quad h_{k}(t, s)=\prod_{v=0}^{k-1} \frac{t-q^{v} s}{\sum_{\mu=0}^{v} q^{\mu}}, \quad \forall t, s \in q^{\mathbb{N}_{0}} .
$$

\section{Some particular perturbed integral inequalities on time scales}

In this section, we point out some particular perturbed integral inequalities on time scales for functions whose second derivatives are bounded as special cases, such as perturbed rectangle inequality on time scales, perturbed trapezoid inequality on time scales, perturbed mid-point inequality on time scales, perturbed Simpson inequality on time scales, perturbed averaged mid-point-trapezoid inequality on time scales, and others. Throughout this section, we always assume that $a, b \in \mathbb{T}$ with $a>b$ and $f:[a, b] \rightarrow \mathbb{R}$ is differentiable. We denote

$$
M=\sup _{a<x<b}\left|f^{\Delta \Delta}(x)\right|<\infty .
$$

Proposition 4.1. Suppose that $\alpha \in[a, b] \cap \mathbb{T}$. Then one has the perturbed rectangle inequality on time scales

$$
\begin{aligned}
& \left|\int_{a}^{b} f^{\sigma}(t) \Delta t-\left((\alpha-a) f^{\sigma}(a)+(b-\alpha) f^{\sigma}(b)\right)+\left(h_{2}(b, \alpha) f^{\Delta}(b)-h_{2}(a, \alpha) f^{\Delta}(a)\right)\right| \\
& \quad \leq M\left(h_{3}(b, \alpha)-h_{3}(a, \alpha)\right) .
\end{aligned}
$$

Proof. We choose $x_{0}=a, x_{1}=b, \alpha_{0}=a, \alpha_{1}=\alpha \in[a, b]$ and $\alpha_{2}=b$ in Theorem 3.1 to get the result.

Remark 4.2. (a) If we choose $\alpha=b$ in (4.2), we get the perturbed left rectangle inequality on time scales

$$
\left|\int_{a}^{b} f^{\sigma}(t) \Delta t-(b-a) f^{\sigma}(a)-h_{2}(a, b) f^{\Delta}(a)\right| \leq-M h_{3}(a, b)
$$


Wenjun Liu et al.

(b) If we choose $\alpha=a$ in (4.2), we get the perturbed right rectangle inequality on time scales

$$
\left|\int_{a}^{b} f^{\sigma}(t) \Delta t-(b-a) f^{\sigma}(b)+h_{2}(b, a) f^{\Delta}(b)\right| \leq M h_{3}(b, a)
$$

(c) If we choose $\alpha=(a+b) / 2$ in (4.2), we get the perturbed trapezoid inequality on time scales

$$
\begin{aligned}
& \left|\int_{a}^{b} f^{\sigma}(t) \Delta t-\frac{f^{\sigma}(a)+f^{\sigma}(b)}{2}(b-a)+\left(h_{2}\left(b, \frac{a+b}{2}\right) f^{\Delta}(b)-h_{2}\left(a, \frac{a+b}{2}\right) f^{\Delta}(a)\right)\right| \\
& \quad \leq M\left(h_{3}\left(b, \frac{a+b}{2}\right)-h_{3}\left(a, \frac{a+b}{2}\right)\right) .
\end{aligned}
$$

Proposition 4.3. Suppose that $x \in[a, b] \cap \mathbb{T}, \alpha_{1} \in[a, x] \cap \mathbb{T}$ and $\alpha_{2} \in[x, b] \cap \mathbb{T}$. Then one has the perturbed inequality on time scales

$$
\begin{aligned}
& \mid \int_{a}^{b} f^{\sigma}(t) \Delta t-\left(\left(\alpha_{1}-a\right) f^{\sigma}(a)+\left(\alpha_{2}-\alpha_{1}\right) f^{\sigma}(x)+\left(b-\alpha_{2}\right) f^{\sigma}(b)\right) \\
& \quad+\left(h_{2}\left(x, \alpha_{1}\right) f^{\Delta}(x)-h_{2}\left(a, \alpha_{1}\right) f^{\Delta}(a)+h_{2}\left(b, \alpha_{2}\right) f^{\Delta}(b)-h_{2}\left(x, \alpha_{2}\right) f^{\Delta}(x)\right) \mid \\
& \quad \leq M\left(h_{3}\left(x, \alpha_{1}\right)-h_{3}\left(a, \alpha_{1}\right)+h_{3}\left(b, \alpha_{2}\right)-h_{3}\left(x, \alpha_{2}\right)\right) .
\end{aligned}
$$

Remark 4.4. If we choose $\alpha_{1}=a$ and $\alpha_{2}=b$ in Proposition 4.3, we get exactly Theorem 1.3. Therefore, Theorem 3.1 is a generalization of Theorem 4 in [20]. If we choose $x=(a+b) / 2$ in (3.1), we get the perturbed mid-point inequality on time scales

$$
\begin{aligned}
& \left|\int_{a}^{b} f^{\sigma}(t) \Delta t-f^{\sigma}\left(\frac{a+b}{2}\right)(b-a)+\left(h_{2}\left(\frac{a+b}{2}, a\right)-h_{2}\left(\frac{a+b}{2}, b\right)\right) f^{\Delta}\left(\frac{a+b}{2}\right)\right| \\
& \quad \leq M\left(h_{3}\left(\frac{a+b}{2}, a\right)-h_{3}\left(\frac{a+b}{2}, b\right)\right) .
\end{aligned}
$$

Corollary 4.5. Suppose that $x \in[(5 a+b) / 6,(a+5 b) / 6] \cap \mathbb{T}, \alpha_{1}=(5 a+b) / 6$ and $\alpha_{2}=(a+5 b) / 6$. Then one has the perturbed inequality on time scales

$$
\begin{aligned}
& \mid \int_{a}^{b} f^{\sigma}(t) \Delta t-\frac{b-a}{3}\left[\frac{f^{\sigma}(a)+f^{\sigma}(b)}{2}+2 f^{\sigma}(x)\right] \\
& \quad+\left(h_{2}\left(x, \frac{5 a+b}{6}\right) f^{\Delta}(x)-h_{2}\left(a, \frac{5 a+b}{6}\right) f^{\Delta}(a)+h_{2}\left(b, \frac{a+5 b}{6}\right) f^{\Delta}(b)-h_{2}\left(x, \frac{a+5 b}{6}\right) f^{\Delta}(x)\right) \mid \\
& \quad \leq M\left(h_{3}\left(x, \frac{5 a+b}{6}\right)-h_{3}\left(a, \frac{5 a+b}{6}\right)+h_{3}\left(b, \frac{a+5 b}{6}\right)-h_{3}\left(x, \frac{a+5 b}{6}\right)\right) .
\end{aligned}
$$


Remark 4.6. If we choose $x=(a+b) / 2$ in (4.8), we get the perturbed Simpson inequality on time scales

$$
\begin{aligned}
& \mid \int_{a}^{b} f^{\sigma}(t) \Delta t-\frac{b-a}{3}\left[\frac{f^{\sigma}(a)+f^{\sigma}(b)}{2}+2 f^{\sigma}\left(\frac{a+b}{2}\right)\right] \\
& +\left[h_{2}\left(\frac{a+b}{2}, \frac{5 a+b}{6}\right) f^{\Delta}\left(\frac{a+b}{2}\right)-h_{2}\left(a, \frac{5 a+b}{6}\right) f^{\Delta}(a)\right. \\
& \left.\quad+h_{2}\left(b, \frac{a+5 b}{6}\right) f^{\Delta}(b)-h_{2}\left(\frac{a+b}{2}, \frac{a+5 b}{6}\right) f^{\Delta}\left(\frac{a+b}{2}\right)\right] \mid \\
& \leq M\left(h_{3}\left(\frac{a+b}{2}, \frac{5 a+b}{6}\right)-h_{3}\left(a, \frac{5 a+b}{6}\right)+h_{3}\left(b, \frac{a+5 b}{6}\right)-h_{3}\left(\frac{a+b}{2}, \frac{a+5 b}{6}\right)\right) .
\end{aligned}
$$

Corollary 4.7. Suppose that $(a+b) / 2 \in \mathbb{T}, \alpha_{1} \in[a,(a+b) / 2] \cap \mathbb{T}$ and $\alpha_{2} \in[(a+b) / 2, b] \cap \mathbb{T}$. Then one has the perturbed inequality on time scales

$$
\begin{aligned}
& \mid \int_{a}^{b} f^{\sigma}(t) \Delta t-\left(\left(\alpha_{1}-a\right) f^{\sigma}(a)+\left(\alpha_{2}-\alpha_{1}\right) f^{\sigma}\left(\frac{a+b}{2}\right)+\left(b-\alpha_{2}\right) f^{\sigma}(b)\right) \\
& \quad+\left(h_{2}\left(\frac{a+b}{2}, \alpha_{1}\right) f^{\Delta}\left(\frac{a+b}{2}\right)-h_{2}\left(a, \alpha_{1}\right) f^{\Delta}(a)+h_{2}\left(b, \alpha_{2}\right) f^{\Delta}(b)-h_{2}\left(\frac{a+b}{2}, \alpha_{2}\right) f^{\Delta}\left(\frac{a+b}{2}\right)\right) \mid \\
& \leq M\left(h_{3}\left(\frac{a+b}{2}, \alpha_{1}\right)-h_{3}\left(a, \alpha_{1}\right)+h_{3}\left(b, \alpha_{2}\right)-h_{3}\left(\frac{a+b}{2}, \alpha_{2}\right)\right) .
\end{aligned}
$$

Remark 4.8. If we choose $\alpha_{1}=(3 a+b) / 4$ and $\alpha_{2}=(a+3 b) / 4$ in (4.10), we get the perturbed averaged mid-point-trapezoid inequality on time scales

$$
\begin{aligned}
& \mid \int_{a}^{b} f^{\sigma}(t) \Delta t-\frac{b-a}{2}\left[\frac{f^{\sigma}(a)+f^{\sigma}(b)}{2}+f^{\sigma}\left(\frac{a+b}{2}\right)\right] \\
& \quad+\left(h_{2}\left(\frac{a+b}{2}, \frac{3 a+b}{4}\right) f^{\Delta}\left(\frac{a+b}{2}\right)-h_{2}\left(a, \frac{3 a+b}{4}\right) f^{\Delta}(a)\right. \\
& \left.\quad+h_{2}\left(b, \frac{a+3 b}{4}\right) f^{\Delta}(b)-h_{2}\left(\frac{a+b}{2}, \frac{a+3 b}{4}\right) f^{\Delta}\left(\frac{a+b}{2}\right)\right) \mid \\
& \leq M\left(h_{3}\left(\frac{a+b}{2}, \frac{3 a+b}{4}\right)-h_{3}\left(a, \frac{3 a+b}{4}\right)+h_{3}\left(b, \frac{a+3 b}{4}\right)-h_{3}\left(\frac{a+b}{2}, \frac{a+3 b}{4}\right)\right) .
\end{aligned}
$$




\section{Acknowledgments}

The authors wish to express their gratitude to the anonymous referees for a number of valuable comments and suggestions. This work was supported by the Science Research Foundation of Nanjing University of Information Science and Technology.

\section{References}

[1] P. Cerone, S. S. Dragomir, and J. Roumeliotis, "An inequality of Ostrowski-Grüss type for twice differentiable mappings and applications in numerical integration," Kyungpook Mathematical Journal, vol. 39, no. 2, pp. 333-341, 1999.

[2] S. S. Dragomir, R. P. Agarwal, and P. Cerone, "On Simpson's inequality and applications," Journal of Inequalities and Applications, vol. 5, no. 6, pp. 533-579, 2000.

[3] S. S. Dragomir, P. Cerone, and J. Roumeliotis, "A new generalization of Ostrowski's integral inequality for mappings whose derivatives are bounded and applications in numerical integration and for special means," Applied Mathematics Letters, vol. 13, no. 1, pp. 19-25, 2000.

[4] W. Liu, Q.-L. Xue, and S.-F. Wang, "Several new perturbed Ostrowski-like type inequalities," Journal of Inequalities in Pure and Applied Mathematics, vol. 8, no. 4, article 110, pp. 1-6, 2007.

[5] W. Liu, C. Li, and Y. Hao, "Further generalization of some double integral inequalities and applications," Acta Mathematica Universitatis Comenianae, vol. 77, no. 1, pp. 147-154, 2008.

[6] W. Liu, "Several error inequalities for a quadrature formula with a parameter and applications," Computers E Mathematics with Applications, vol. 56, no. 7, pp. 1766-1772, 2008.

[7] D. S. Mitrinović, J. E. Pečarić, and A. M. Fink, Inequalities for Functions and Their Integrals and Derivatives, Kluwer Academic Publishers, Dordrecht, The Netherlands, 1994.

[8] D. S. Mitrinović, J. E. Pečarić, and A. M. Fink, Classical and New Inequalities in Analysis, vol. 61 of Mathematics and Its Applications, Kluwer Academic Publishers, Dordrecht, The Netherlands, 1993.

[9] N. Ujević, "A generalization of Ostrowski's inequality and applications in numerical integration," Applied Mathematics Letters, vol. 17, no. 2, pp. 133-137, 2004.

[10] S. Hilger, Ein maßkettenkalkül mit anwendung auf zentrumsmannigfaltigkeiten, Ph.D. thesis, Universitaat Würzburg, Würzburg, Germany, 1988.

[11] R. Agarwal, M. Bohner, and A. Peterson, "Inequalities on time scales: a survey," Mathematical Inequalities $\mathcal{E}$ Applications, vol. 4, no. 4, pp. 535-557, 2001.

[12] M. R. S. Ammi, R. A. C. Ferreira, and D. F. M. Torres, "Diamon- $\alpha$ Jensen's inequality on time scales," Journal of Inequalities and Applications, vol. 2008, Article ID 576876, 13 pages, 2008.

[13] M. Bohner, S. Clark, and J. Ridenhour, "Lyapunov inequalities for time scales," Journal of Inequalities and Applications, vol. 7, no. 1, pp. 61-77, 2002.

[14] M. Bohner and T. Matthews, "The Grüss inequality on time scales," Communications in Mathematical Analysis, vol. 3, no. 1, pp. 1-8, 2007.

[15] M. Bohner and T. Matthews, "Ostrowski inequalities on time scales," Journal of Inequalities in Pure and Applied Mathematics, vol. 9, no. 1, article 6, pp. 1-8, 2008.

[16] W. N. Li and W. Sheng, "Some nonlinear integral inequalities on time scales," Journal of Inequalities and Applications, vol. 2007, Article ID 70465, 15 pages, 2007.

[17] F.-H. Wong, S.-L. Yu, and C.-C. Yeh, "Anderson's inequality on time scales," Applied Mathematics Letters, vol. 19, no. 9, pp. 931-935, 2006.

[18] C.-C. Yeh, H.-L. Hong, H.-J. Li, and S.-L. Yu, "Some complements of Cauchy's inequality on time scales," Journal of Inequalities and Applications, vol. 2006, Article ID 97430, 19 pages, 2006.

[19] W. Liu and Q.-A. Ngô, "A generalization of Ostrowski inequality on time scales for $k$ points," Applied Mathematics and Computation, vol. 203, no. 2, pp. 754-760, 2008.

[20] W. Liu and Q.-A. Ngô, "An Ostrowski type inequality on time scales for functions whose second derivatives are bounded," to appear in Inequality Theory and Applications, vol. 6, 2009.

[21] M. Bohner and A. Peterson, Dynamic Equations on Time Scales: An Introduction with Application, Birkhäuser, Boston, Mass, USA, 2001. 
[22] M. Bohner and A. Peterso, Eds., Advances in Dynamic Equations on Time Scales, Birkhäuser, Boston, Mass, USA, 2003.

[23] V. Lakshmikantham, S. Sivasundaram, and B. Kaymakcalan, Dynamic Systems on Measure Chains, vol. 370 of Mathematics and Its Applications, Kluwer Academic Publishers, Dordrecht, The Netherlands, 1996.

[24] A. Sofo and S. S. Dragomir, "A perturbed version of the Ostrowski inequality for twice differentiable mappings," Turkish Journal of Mathematics, vol. 25, no. 3, pp. 379-412, 2001. 